\title{
Application of Multi-sensor Information Fusion Technology on Fault Diagnosis of Electrical System
}

\author{
Ling-Wen MENG ${ }^{1, a}$, Ji-Pu GAO ${ }^{1}$, Ming-Yong XIN ${ }^{1}$, Jin-Mei XIONG ${ }^{2}$ and Rui GUO ${ }^{2}$ \\ ${ }^{1}$ Guizhou Electric Power Research Institute of Guizhou Power Grid Co., Ltd., Guiyang, 550002, Guizhou, China \\ ${ }^{2}$ Wuhan Zhongyuan Huadian Science \& Technology Co., Ltd, Wuhan 430074, China
}

\begin{abstract}
This paper presents a multi-sensor information fusion technology based on electrical system fault diagnosis model, analysis the multi-sensor information fusion technology of several structure layer and realization method, and discusses its application in fault diagnosis of electrical system. Select and implement appropriate methods for the information fusion, the different error characteristic parameters in electrical system can be fused and calculated by this model, and a more valuable conclusion for the fault diagnosis of electrical system can be provided.
\end{abstract}

\section{Introduction}

Electricity plays a vital role in human life. Fault diagnosis need to ensure that the electrical system is safe and reliable, without any potential accidents. However, in practice, whether the system is faulty can't be determined by diagnosis based on one parameter. But different angles of the electrical system operating parameters can be gotten by the multi-sensor information fusion technology, Thereby effectively integrating and merging all the parameters. Not only Fault diagnosis of electrical system can be guaranteed, but also identify and locate the electrical system failure accurately. This paper analyzes the multi-sensor information fusion technology and its application in the electrical system.

\section{Technology of multi-sensor Information Fusion}

Multi-sensor information fusion is a comprehensive multi-layer processing program. In order to realize the state estimation, including the situation estimation of the system and the risk estimation of the system, the system has the functions of detecting, predicting, merging and combining all the parameters measured in the electrical system.[1,2] The fault diagnosis system which is designed for the power system using multi-sensor information fusion technology, this technology including data fusion and knowledge fusion, moreover, data-knowledge fusion, namely data mining.

\subsection{Layers of multi-sensor information fusion}

As shown in Figure 1, information fusion can be divided into 3 layers [3].

\footnotetext{
${ }^{\mathrm{a}}$ Corresponding author: 1205954738@qq.com
} 


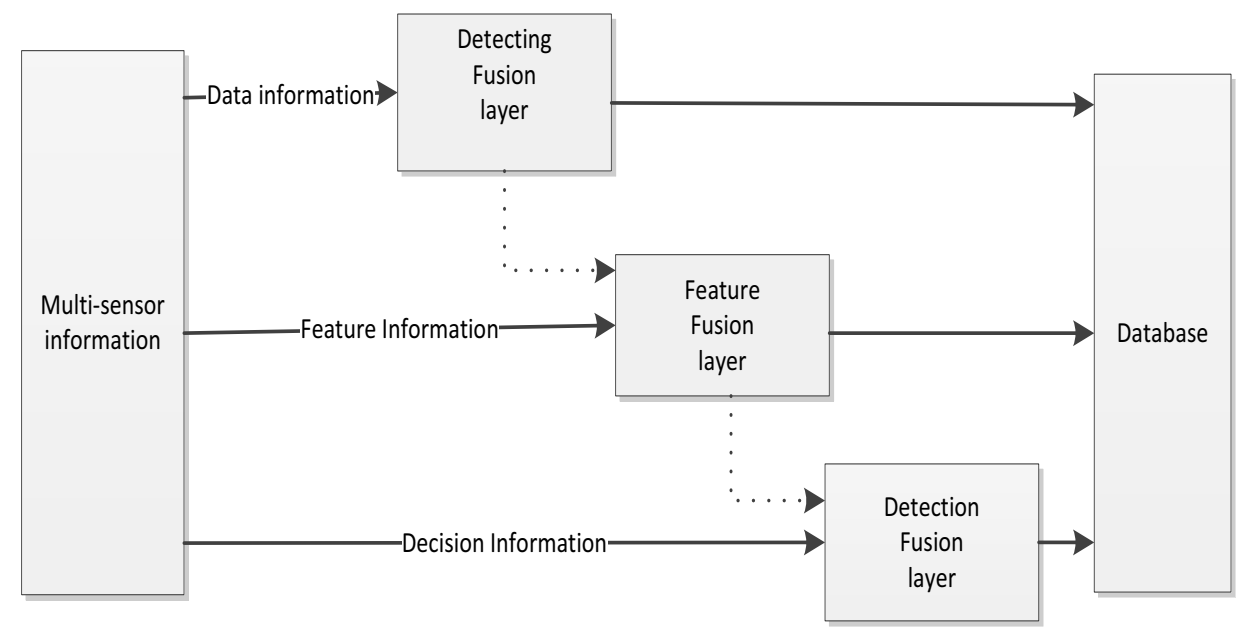

Figure 1. Caption of the Figure 1. Below the figure.

- Detection Layer: Primarily responsible for the fusion of the original information measured by the same type of sensor before preprocessing. So in the shortest time, the system operating conditions can be intuitively and sensitively detect the information. At the same time all the information is entered into the database for data mining. This is the prerequisite for fault diagnosis.

- Feature layer: Mainly responsible for the integration of the original information and related theoretical knowledge measured various sensors. In this way, it can accurately identify and locate faults in the electrical system. Thus, to ensure that when the system fails, the staff can know the cause of the malfunction in the shortest time. [4, 5]

- Decision layer: This is the highest layer in the system. System fuse all the information and related theoretical knowledge which is measured by different kinds of sensors, in order to achieve including fault isolation, redundant control for specific methods and techniques of fault diagnosis. And if the final countermeasure is feasible, the experience of such fault will be entered into the database for use at a certain time.

\subsection{Methods of multi-sensor information fusion}

In the variety of sensor information fusion methods, such as Bayesian theory, Damper Shafer (D-S) theory, neural network technology and some estimation theory and so on, as a modification theory of Bayes' theorem, D-S theory has more extensive application in multi-sensor information fusion technology. The method is able to display the determined probabilities and the uncertain probabilities of the information, and eliminates the simple assumptions about the unknown probabilities.

The evidence is set into mutually independent parts by the basic way of D-S theory. Every evidence part has a belief function which means probability distribution function to the related theoretical diagnosis. The total belief degree of comprehensive evidence based on related theoretical knowledge is obtained by fusion based on different clues and diagnosis of related theoretical knowledge.[6-8] Figure 2 shows the course of reasoning of D-S theory. 


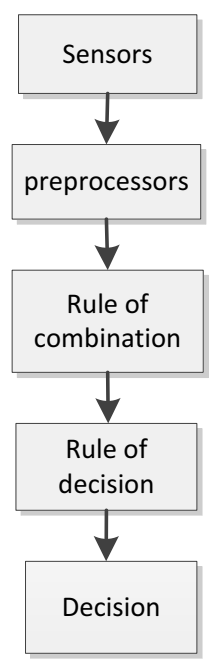

Figure 2. Schematic diagram of the course of reasoning of D-S theory

\section{Application of multi-sensor information fusion technology on fault diagnosis of hydraulic system}

\subsection{Structure and principle of the fault diagnosis system}

The electrical system common failure consist of include leakage, wire wear, corrosion, equipment fatigue and so on. The electrical system based on multi-sensor information fusion technology should monitor parameters which include power system voltage, current flow, equipment temperature, oil spills and so on. Detection and diagnosis system mainly includes two functional modules: data acquisition module and central process module. The data acquisition module includes sensors, signal conditioning circuits, A / D converters and bus interfaces, which are installed in each important part to collect and transmit each status signal of the power system. Central processing module Implement data analysis, fault diagnosis, integration and give response methods and other functions. It is mainly composed of CPU and computer software. The fault data of the electrical system is stored in the database[9].

Figure 3 shows the block diagram of the model of fault diagnosis system. Figure 4 shows the flow chart of the diagnostic program. 


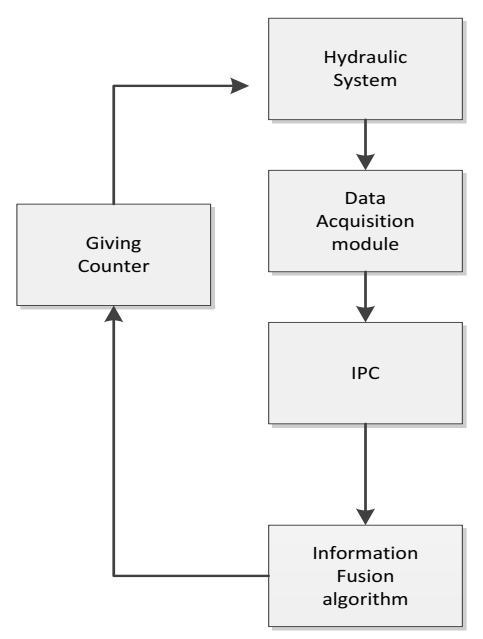

Figure 3. Block diagram of the model of fault diagnosis

\subsection{Characteristics of the fault diagnosis system}

- The system can monitor all the operating parameters at any time, including voltage, current, temperature, oil leakage.

- All operational parameters measured by the sensor are fused by the D-S theory .Not only can successfully achieve state recognition, but also for some typical failure to make a diagnosis and security protection.

\subsection{Key technology of the fault diagnosis system}

- Getting accurate signals id very important. It is critical to select the type of sensor reasonably and preprocess all available signals.

- The method of selecting and realizing information fusion is the guarantee of fault diagnosis. The D-S theory approach may not achieve the desired results under certain conditions, thus affecting the stability of the system. So neural network technology may be a better choice. 


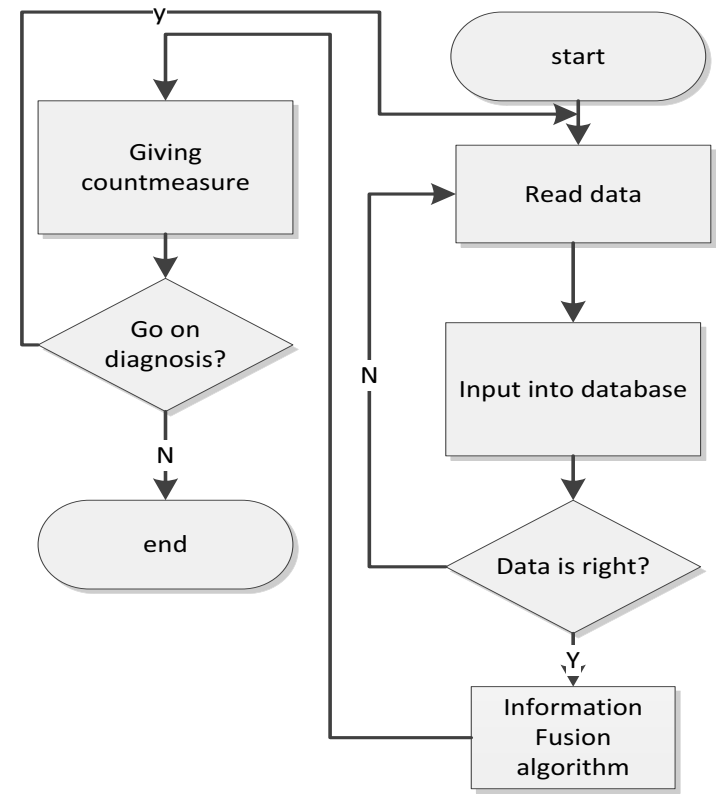

Figure 4. Flow chart of the diagnostic program

\section{Conclusions}

This paper presents a fault diagnosis system based on multi-sensor information fusion technology. The system realizes the status alarm and diagnosis of the electrical system. By making full use of a plurality of signals that can be measured from the power system, information fusion is realized, and the system is diagnosed in time. This can improve the efficiency and safety of the power system. This fault diagnosis system model based on electrical system described in this paper is a generalized model. In practice, for different power systems, fault diagnosis system monitoring parameters and the actual structure is different.

\section{References}

1. Jun Han, Jianzhong Zhang, Ming Cheng. Fault diagnosis of wind turbine based on multi- sensors information fusion technology, IET renewable power generation. 8(3) (2014)

2. Fengyong Lang,Xiaogang Li . Multi-Sensors Information Fusion Based on Momentis Method and Euclid Distance, International Conference on Manufacturing Science and Technology. (2011)

3. Fengyong Lang,Xiaogang Li. Multi-Sensors Information Fusion Based on Momentis Method and Euclid Distance. International Conference on Manufacturing Science and Technology (ICMST) (2012)

4. Halld L. Mathematical technique in multi-sensor data fusion Artech House 15-21 (2000)

5. H F. Durrant Whyte Sensor models and multi-sensor integration. The Int. J. of Robotics Research 7(6) 87-92(2011)

6. Mourad O. Some notes on fusion of uncertainty information. Int. J. of Intelligent Systems 19(6) 457-471(2012)

7. Richard T. Principles of effective multisensor data fusion, Military Technology 27(5) 2937.(2013) 
8. An F Y, Lu H W, Liu C J, et al. Research and application of information fusion technology on machinery fault diagnosis Chinese Journal of Chongqing University 29(1) 15-18 (2006)

9. Ge Bing, Yu Yi. Application of Multi-Sensors Data Fusion Technology in the Theodolite Tracking system. International Conference on Mechanical and Electrical Technology 5th. (2013) 